\title{
Wide-scope screening of pharmaceuticals, illicit drugs and their metabolites in the Amazon River
}

\author{
David Fabregat-Safonta ${ }^{a}$ María Ibáñez ${ }^{a}$, Lubertus Bijlsma a, Félix Hernández ${ }^{a}$, \\ Andrea V. Waichman ${ }^{b}$, Rhaul de Oliveira ${ }^{c}$, Andreu Rico ${ }^{\mathrm{d}, \mathrm{e}, *}$ \\ a Environmental and Public Health Analytical Chemistry, Research Institute for Pesticides and Water (IUPA), University Jaume I, Avda. Sos Baynat s/n, 12071, \\ Castellón, Spain \\ ${ }^{\mathrm{b}}$ Federal University of the Amazon, Institute of Biological Sciences, Av. Rodrigo Otávio Jordão Ramos 3000, Manaus 69077-000, Brazil \\ ' University of Campinas, School of Technology, Rua Paschoal Marmo 1888 - Jd. Nova Itália, Limeira 13484-332, Brazil \\ d IMDEA Water Institute, Science and Technology Campus of the University of Alcalá, Av. Punto Com 2, Alcalá de Henares 28805, Madrid, Spain \\ e Cavanilles Institute of Biodiversity and Evolutionary Biology, University of Valencia, c/ Catedrático José Beltrán 2, 46980, Paterna, Valencia, Spain
}

\section{A R T I C L E I N F O}

\section{Article history:}

Received 30 March 2021

Revised 29 April 2021

Accepted 11 May 2021

Available online 17 May 2021

\section{Keywords:}

Pharmaceuticals

Freshwater ecosystems

Ion mobility

High-resolution mass spectrometry

Screening

Environmental monitoring

\begin{abstract}
A B S T R A C T
Only a limited number of households in the Amazon are served by sewage collection or treatment facilities, suggesting that there might be a significant emission of pharmaceuticals and other wastewater contaminants into freshwater ecosystems. In this work, we performed a wide-scope screening to assess the occurrence of pharmaceuticals, illicit drugs and their metabolites in freshwater ecosystems of the Brazilian Amazon. Our study included 40 samples taken along the Amazon River, in three of its major tributaries, and in small tributaries crossing four important urban areas (Manaus, Santarém, Macapá, Belém). More than 900 compounds were investigated making use of target and suspect screening approaches, based on liquid chromatography coupled to high-resolution mass spectrometry with ion mobility separation. Empirical collision-cross section (CCS) values were used to help and confirm identifications in target screening, while in the suspect screening approach CCS values were predicted using Artificial Neural Networks to increase the confidence of the tentative identification. In this way, 51 compounds and metabolites were identified. The highest prevalence was found in streams crossing the urban areas of Manaus, Macapá and Belém, with some samples containing up to 30 - 40 compounds, while samples taken in Santarém showed a lower number $(8-11)$, and the samples taken in the main course of the Amazon River and its tributaries contained between 1 and 7 compounds. Most compounds identified in areas with significant urban impact belonged to the analgesics and antihypertensive categories, followed by stimulants and antibiotics. Compounds such as caffeine, cocaine and its metabolite benzoylecgonine, and cotinine (the metabolite of nicotine), were also detected in areas with relatively low anthropogenic impact and showed the highest total prevalence. This study supports the need to improve the sanitation system of urban areas in the Brazilian Amazon and the development of follow-up studies aimed at quantifying exposure levels and risks for Amazonian freshwater biodiversity.
\end{abstract}

(c) 2021 Elsevier Ltd. All rights reserved.

\section{Introduction}

The Amazon is the largest drainage basin in the world and contains about $40 \%$ of the world's remaining tropical rainforest. It plays a crucial role in maintaining global hydrology and climate, and hosts about $25 \%$ of the global freshwater biodiversity (Oberdorff et al., 2019; Tedesco et al., 2017; Tisseuil et al., 2013). The Amazonian freshwater ecosystems are threatened by

* Corresponding author at: IMDEA Water Institute, Avenida Punto Com 2, 28805 Alcalá de Henares, Spain.

E-mail address: andreu.rico@imdea.org (A. Rico). land use changes and habitat fragmentation related to several anthropogenic pressures, such as mining, deforestation, damming or agricultural expansion (Castello and Macedo, 2016; Jézéquel et al., 2020). One of the less investigated, although relevant, anthropogenic pressures over Amazonian ecosystems is urbanization. Today, about $80 \%$ of the Amazonian population live in cities, including the metropolitan areas of Manaus and Belém, with more than 2.5 million inhabitants each (IBGE, 2020). Urbanization contributes to air, soil, and freshwater contamination (Ferreira et al., 2021; Puppim de Oliveira et al., 2011; Shrivastava et al., 2019). Freshwater contamination in the Amazon is of critical relevance since more than $90 \%$ of households in the region are not served by any sewage 
collection or treatment facilities (SNIS, 2020), and discharge tons of solid and liquid waste directly into the river network. In fact, organic pollutants such as polycyclic aromatic hydrocarbons (dos Santos Rodrigues et al. 2018), plastic additives (Schmidt et al., 2019), or pharmaceuticals, illicit drugs and personal care products (Chaves et al., 2020; Thomas et al., 2014) have been reported in water and sediment samples collected in the Amazon Estuary and in water bodies with significant anthropogenic pressure. One of the main problems of freshwater contamination is that it tends to spread throughout the river basin, reaching protected areas of high ecological value (Saunders et al., 2002). Among them, floodplains are of utmost importance, since they are key in sustaining fish reproduction and provide food resources for local populations (Hurd et al., 2016; Begossi et al., 2019). Therefore, the protection of Amazonian freshwater ecosystems requires the understanding of the main contaminants that are currently emitted into the river network and their distribution in relation to their major emission points.

Advanced analytical methodologies allow the identification of a wide variety of contaminants in environmental samples. Mass spectrometry (MS) coupled to gas (Canlı et al., 2020; Sotão Neto et al., 2020) and liquid chromatography (Fonseca et al., 2019; Tian et al., 2020) are nowadays the gold-standards for the identification and quantification of organic contaminants in environmental samples (Pérez and Barceló, 2007). In the last few years, Ultra-High Performance Liquid chromatography (UHPLC) coupled to tandem MS (MS/MS), or to high-resolution MS (HRMS), have been widely used for accurate quantification of organic micropollutants (Boix et al., 2014; Gracia-Lor et al., 2011), or for widescope screening (Guardian et al., 2021; Hernández et al., 2015b, 2015a; Llorca et al., 2021; Lotfi Khatoonabadi et al., 2021), respectively. The latter allows the full-data acquisition of all the ionizable compounds present in the sample, enabling the possibility to perform target, suspect or non-target screening, also in a retrospective way (Choi et al., 2021; Hollender et al., 2017; Menger et al., 2020). The accurate-mass full-spectrum acquisition is highly valuable for the identification of suspect compounds, such as metabolites and transformation products, as there are not analytical reference standards available for many of them, and therefore identification must be based on accurate mass fragmentation (Boix et al., 2016a, 2016b), among other MS data. UHPLC-HRMS provides a large amount of complex analytical data; therefore, appropriate workflows are needed for compounds identification based on chromatographic separation, accurate-mass measurements and fragmentation information (Schymanski et al., 2014). In the last few years, the use of HRMS with ion mobility separation (IMS-HRMS) has significantly increased in different research fields, such as the analysis of natural compounds or food safety (Canellas et al., 2019). However, IMS has been less explored in environmental analysis (Hinnenkamp et al., 2019). LC-IMS-HRMS provides an extra identification parameter, in addition to chromatographic retention time and accurate mass. The collision cross-section (CCS) value provided by IMS and derived from the drift time is unique and unaffected by the matrix or chromatographic separation. Experimental CCS values have been proven useful in identifying various target compounds. Moreover, in wide-scope screening approaches, the utilization of predictive CCS models facilitate and give more reliability to the tentative identification of suspect compounds (Bijlsma et al., 2017, 2019; Mullin et al., 2020). In front of this scenario, a refined workflow for target and suspect environmental analysis using IMSHRMS data has been proposed (Celma et al.,2020), including complementary identification levels criteria for IMS and updating the criteria previously reported on confidence levels (Schymanski et al., 2014).

In this work, a combined target and suspect UHPLC-IMS-HRMS screening has been applied for the identification of pharmaceu- ticals, illicit drugs and their metabolites in water samples collected from 40 sampling sites in the Amazon River network, which have different level of anthropogenic impact. To facilitate suspect screening analyses, a data processing procedure has been proposed filtering the candidates based on different LC-IMS-HRMS parameters, including predicted CCS values. Therefore, the objectives of the present work were: (i) to implement a novel UHPLC-IMS-HRMS data processing workflow for identification of suspect compounds, facilitating data treatment; (ii) to identify pharmaceuticals and related products in the Amazon River water samples by the application of an advanced target and suspect screening strategy based on UHPLC-IMS-HRMS; (iii) to evaluate the obtained results as regards to different levels of urban pressure.

\section{Materials and methods}

\subsection{Chemicals and materials}

HPLC-grade water was obtained by purifying demineralized water using a Milli-Q system from Millipore (Bedford, MA, USA). LCMS grade methanol, LC-MS grade acetonitrile, and LC-MS grade formic acid were purchased from Scharlau (Scharlab, Barcelona, Spain).

\subsection{Amazon River water samples}

Surface water samples $(n=40)$ were collected between November $16^{\text {th }}$ and December $8^{\text {th }}$ of 2019 , during the low water season, from different locations of the Brazilian Amazon (Fig. 1). Samples were taken from the Amazon River (upper and lower reach, I and II, respectively, $\mathrm{n}=11$ ), from three major tributaries (Negro River $(\mathrm{n}=5)$, Tapajos River $(\mathrm{n}=2)$ and Tocantins Rivers $(\mathrm{n}=2))$, and from smaller tributaries and streams crossing the urban areas of Manaus $(n=8)$, Santarém $(n=3)$, Macapá $(n=3)$ and Belém $(n=6)$. The samples in the Negro River included two locations in the Anavilhanas National Park (N1 and N2), which is a relatively pristine area. Most samples from the Amazon River were collected relatively close to small urban areas, while some samples from the main tributaries were taken near the discharge area of major cities. For example, samples from the Negro River (N4 and N5) correspond to the dilution area of Manaus, while the sample TO2, taken in the Tocantins River, was collected downstream of Belém. Further details on the sampling sites, such as sampling date, GPS coordinates or name of the stream/river, are provided in the Supplementary Information file (Table S1). Grab sampling was done from boats or urban bridges by using a pre-washed metal bucket and collecting water from a depth of approximately $20-30 \mathrm{~cm}$. Water samples $(2 \mathrm{~L})$ were introduced into amber glass bottles and stored at $-4^{\circ} \mathrm{C}$ (under dark conditions) for a maximum of $48 \mathrm{~h}$ until extraction.

\subsection{Sample treatment}

Water samples were pre-filtered through a $0.7 \mu \mathrm{m}$ glass fibber filter (Merck Millipore, Cork, IRL) and subjected to solid-phase extraction (SPE). For this, the sample $\mathrm{pH}$ was adjusted to 8 - 9 by adding few drops of $\mathrm{NH}_{4} \mathrm{OH}$ at $32 \%$. Then, SPE cartridges (Oasis HLB, $200 \mathrm{mg} / 6 \mathrm{cc}$, Waters, Mildford, MA, USA) were preconditioned with $6 \mathrm{~mL}$ of methanol, $6 \mathrm{~mL}$ of ultrapure water and $6 \mathrm{~mL}$ of ultrapure water at basic $\mathrm{pH}(8-9)$. The water samples $(100 \mathrm{~mL})$ were passed through the SPE cartridges using a vacuum manifold, rinsed with $10 \mathrm{~mL}$ of ultrapure water, and dried for $10 \mathrm{~min}$. The loaded SPE cartridges were properly labelled, sealed and shipped at $-20^{\circ} \mathrm{C}$ to the Spanish laboratories. Afterwards, the SPE cartridges were eluted with methanol (three aliquots of $4 \mathrm{~mL}$ ). The extracts were evaporated to dryness at $45^{\circ} \mathrm{C}, 0.2$ Torr using a SpeedVac 


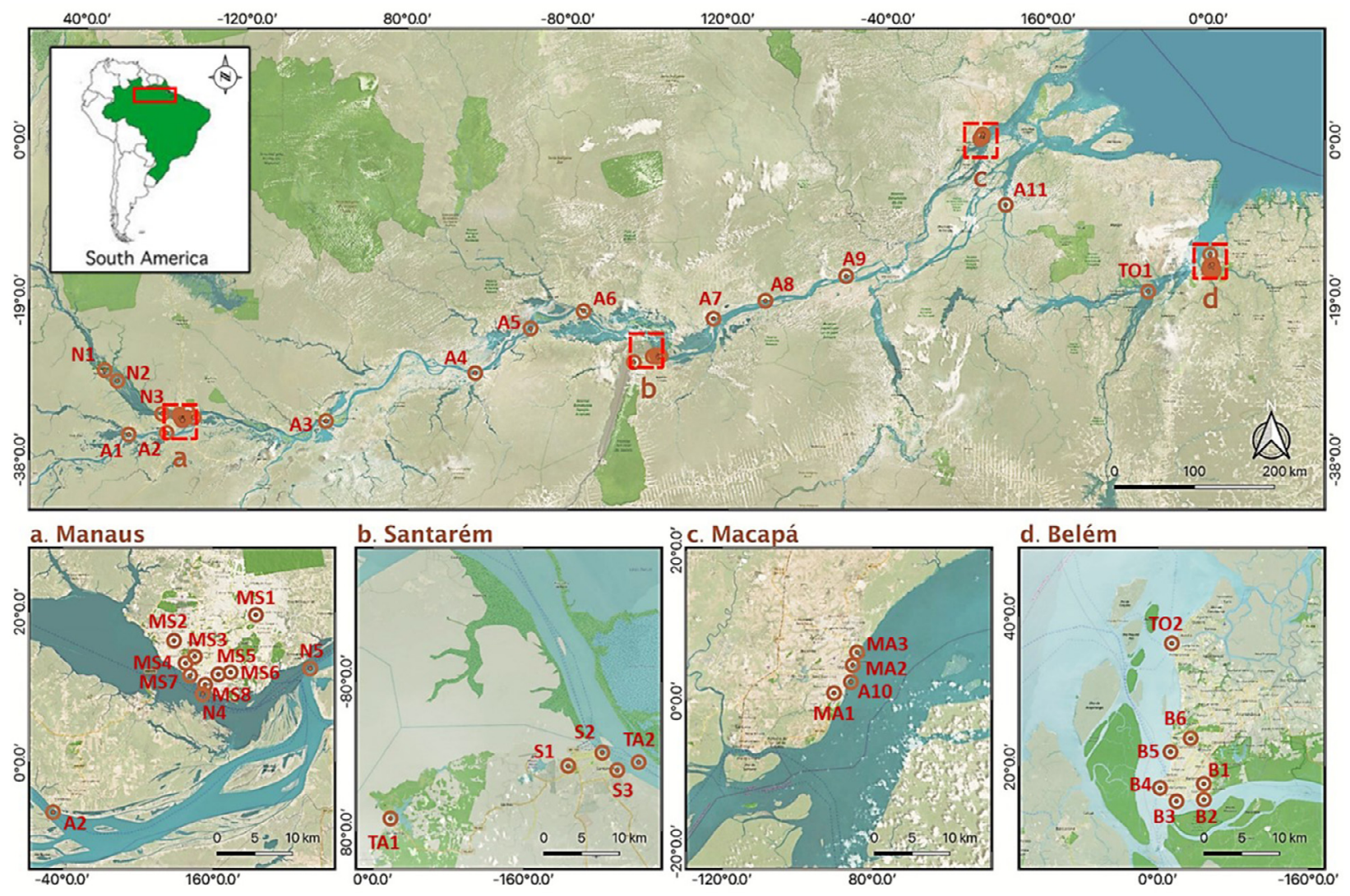

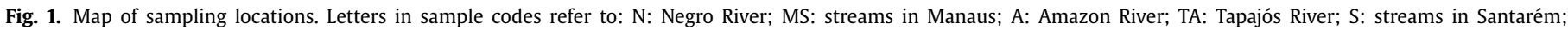
MA: streams in Macapá; TO: Tocantins River; B: streams in Belém.

concentrator (Thermo Scientific, Massachusetts, USA), reconstituted with $1 \mathrm{~mL}$ of methanol:water (10:90, v/v), and vortex stirring for 1 min. Finally, they were centrifuged for $5 \mathrm{~min}$ at 13,000 rpm (MiniSpin centrifuge, Eppendorf, USA) and transferred into amber glass vials.

\subsection{Instrumentation}

Samples were analyzed using a Waters Acquity I-Class UPLC system (Waters Corp., Milford, MA, USA) coupled to a Vion IMS QTOF mass spectrometer (Waters Corp., Wilmslow, Manchester, $\mathrm{UK}$ ), using an electrospray ionization (ESI) interface operating in both positive and negative ionization modes.

The chromatographic separation was performed using a Cortecs C18 $2.1 \times 100 \mathrm{~mm}, 2.7 \mu \mathrm{m}$ fused core column (Waters Corp., Wex-

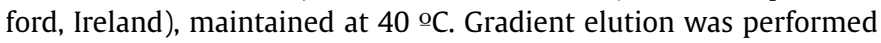
at a flow rate of $0.3 \mathrm{~mL} / \mathrm{min}$, using water (A) and methanol (B) both with $0.01 \%$ formic acid, changing as follows: $10 \% \mathrm{~B}$ at 0.0 min, $90 \% \mathrm{~B}$ at $14.0 \mathrm{~min}, 99 \% \mathrm{~B}$ at $14.1 \mathrm{~min}, 99 \% \mathrm{~B}$ at $16.0 \mathrm{~min}$, $10 \% \mathrm{~B}$ at $16.1 \mathrm{~min}$, with a total run time of $18 \mathrm{~min}$. The volume injection was $5 \mu \mathrm{L}$.

The ESI was operated with a capillary voltage of $1.0 \mathrm{kV}$ in $\mathrm{ESI}^{+}$ and $1.5 \mathrm{kV}$ in $\mathrm{ESI}^{-}$, using in both cases a cone voltage of $30 \mathrm{~V}$. The source temperature was set to $120 \stackrel{\circ}{ }$, while the desolvation temperature at $650 \circ \mathrm{C}$, using nitrogen as desolvation gas at 1200 $\mathrm{L} / \mathrm{h}$ and cone gas at $250 \mathrm{~L} / \mathrm{h}$. Nitrogen ( $\geq 99.999 \%$ ) was used as drift gas, with an IMS wave velocity of $250 \mathrm{~m} / \mathrm{s}$ and wave height ramp of $20-50 \mathrm{~V}$. The TOF resolution was $\sim 36,000$ FWHM $(\mathrm{m} / \mathrm{z}$ $556)$ in positive ionization mode and $\sim 38,000$ FWHM $(\mathrm{m} / z$ 554) in negative. Calibration was performed using the "major mix IMS/TOF calibration solution" (Waters Corp) following manufacturer's recommendations. Leucine enkephalin $(100 \mu \mathrm{g} / \mathrm{L}$ in water:acetonitrile
50:50 containing $0.01 \%$ of formic acid) was used for continuous mass correction during all chromatographic run. Two independent scan functions were acquired sequentially: a low-energy (LE) function using a collision energy of $6 \mathrm{eV}$, and a high-energy (HE) using a ramp of 28-56 eV for high energy (HE). Nitrogen ( $\geq 99.999$ $\%$ ) was used as collision-induced dissociation (CID) gas. Both functions were acquired in the range of $m / z 50-1000$ with a scan time of $0.3 \mathrm{~s}$. Data were acquired and processed using the UNIFI informatics platform ( $\mathrm{v}$ 1.9) from Waters.

\subsection{Screening strategy}

The wide-scope screening applied for identification of illicit drugs, pharmaceuticals and their metabolites in Amazon River samples was performed using an in-house built compound database (available in Fonseca et al., 2020). This database contained information about the chromatographic retention time (RT), CCS value, and elemental composition (including fragment ions) of 290 target compounds. For the remaining ones (631 suspect compounds, including most of the metabolites), only information about the elemental composition was available. When available, fragment ions previously reported in the literature were also included. Two different data treatment procedures were employed, one for target screening and another one for suspect screening (Fig. 2). In both cases, compound identification was based on the 5-confidence levels recently proposed for IMS-HRMS-based methods, including criteria for the CCS parameter (Celma et al., 2020).

\subsubsection{Target screening analysis}

A database built with 290 reference standards was used, including pharmaceuticals, illicit drugs, and several of their main 


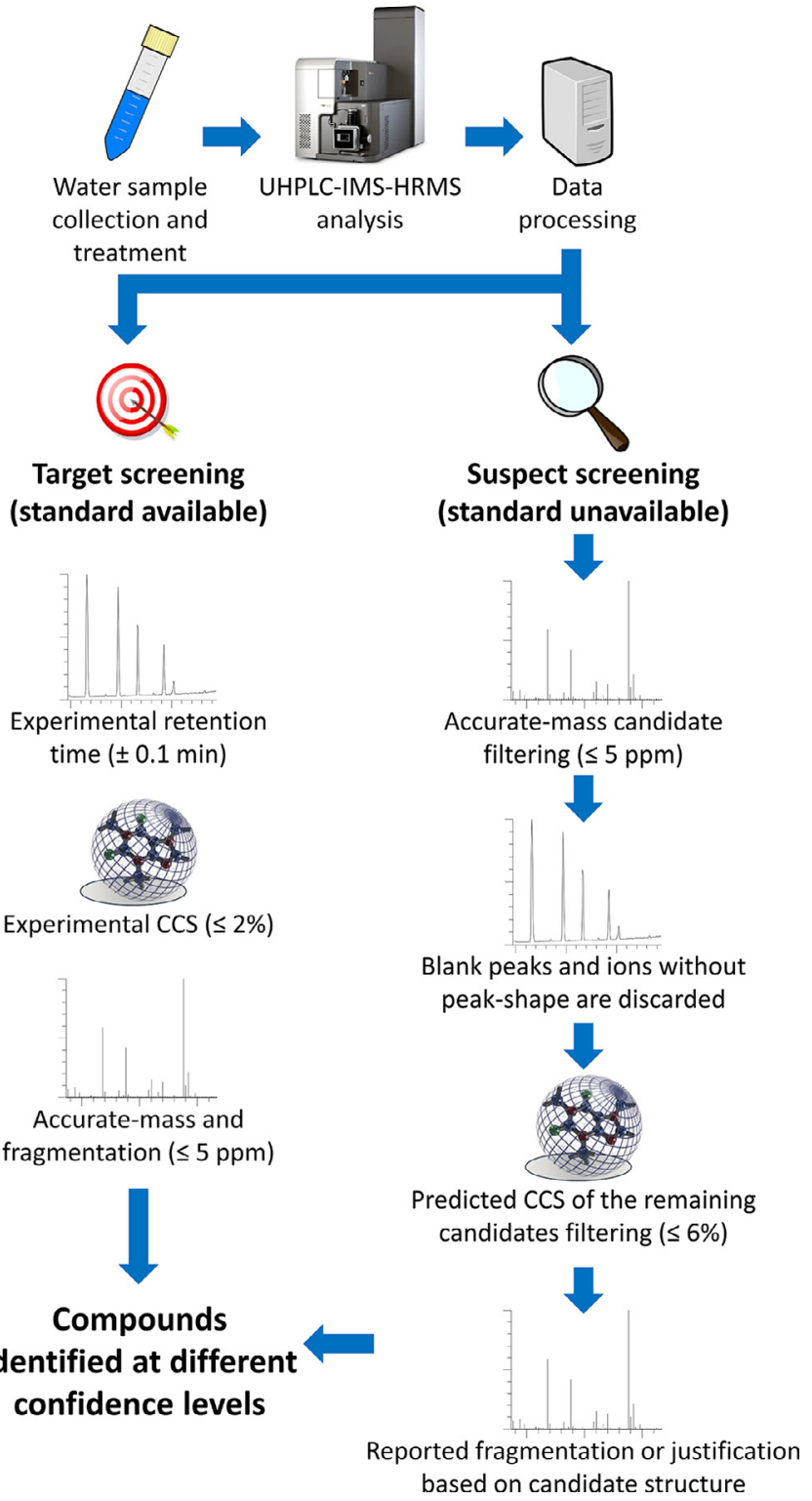

Fig. 2. Data processing workflow for UHPLC-IMS-HRMS target and suspect screening.

metabolites (Celma et al., 2020). Compound filtering was performed based on experimental RT, CCS, mass accuracy and fragmentation. Briefly, a compound confirmed with the highest reliability (Level 1) should present a chromatographic RT deviation lower than $0.1 \mathrm{~min}$, CCS deviation $\leq 2 \%$, mass error lower than $5 \mathrm{ppm}$ for the (de)protonated molecule, and at least 1 fragment ion being observed. If one of these requirements was not achieved, the compound was considered as identified at Level 2 . In absence of a reference standard, isotope pattern and fragment ions previously reported for these compounds (in literature or MS spectra databases) were used for identification, as well as RT and CCS values from databases or predicted from computational models (Bade et al., 2015; Bijlsma et al., 2017).

\subsubsection{Suspect screening analysis}

For suspect screening, an in-house database containing 631 compounds was used, including pharmaceuticals, hormones, illicit drugs, as well as metabolites and transformation products previously found in water samples. For the suspect compounds, no analytical reference standards were available at our laboratory, while information about their fragmentation was only available for 85 of them.

To simplify data evaluation, a three-step workflow was applied including compound filtering by accurate mass, chromatographic peak shape and predicted CCS values, with the aim to reduce the number of false positives. The first filtering was based on the accurate mass of the ions detected in the LE function (typically the (de)protonated molecule), focusing the subsequent tentative identification on those compounds that presented a mass error lower than $5 \mathrm{ppm}$ with respect to the theoretical exact mass. Then, those candidates also detected in procedural blanks or those that did not present appropriate peak-shape in the chromatogram were discarded. In a second step, CCS values were predicted using Artificial Neural Networks (Bijlsma et al., 2017) for the remaining compounds, and candidates were filtered based on experimental CCS, considering only those compounds with a deviation below $6 \%$ respect to the predicted value (95\% confidence interval). In the last step, the accurate mass fragments were justified based on the candidate structure. In the case of metabolites, the presence of common fragments shared with the unaltered compounds was also used as an identification parameter. Fig. 2 shows a schematic representation of the screening procedure applied. Further details on data processing can be found in the Supplementary Information.

\section{Results and discussion}

The combination of target and suspect screening using HRMS is nowadays one of the most powerful approaches for screening a large number of organic micropollutants in water. The complementary use of gas and liquid chromatography coupled to HRMS, together with a sample treatment based on SPE with a polymericbased cartridge, such as Oasis HLB, has been described as one of the most universal approaches to that aim (Hernandez et al, 2015b). However, a "true universal" method does not exist, as there are always a number of compounds that can be lost along the process (e.g. highly polar compounds). Thus, the previous SPE treatment applied in this work can be a limiting step, as some compounds may be not retained and/or not eluted from the cartridge. In addition, some compounds cannot be ionized in the ESI interfaces of LC-MS, and persistent organic pollutants, of low polarity, such as many organochlorine compounds, do not fit well with LCMS based analysis. Despite these limitations, the methodology applied in this work provides a realistic overview of the occurrence of about 1,000 organic micro-pollutants in Amazonian waters, taking into account that the majority of pharmaceuticals, illicit drugs and metabolites are commonly analyzed by LC-MS.

\subsection{Target screening results}

Table 1 shows the compounds identified in water samples by target screening. Most of the detected compounds were identified at the confidence Level 1. However, in some samples only Level 2 could be reached, a fact that could be explained by the differences in the water matrix samples and/or in their contamination levels. Thus, samples collected in streams near the main urban areas commonly presented much higher MS signals for the compounds under study, which notably facilitated their identification.

For several compounds identified at Level 2, the detection in both positive and negative ionization modes increased the confidence. This was the case of acetaminophen and sucralose. For the later, the typical isotope pattern of the three chlorine atoms was observed in $\mathrm{ESI}^{+}$(as sodium adduct, Fig. 3A) and $\mathrm{ESI}^{-}$(as deprotonated molecule, Fig. 3B). Sucralose presents poor fragmentation in ESI-, as shown in the MS spectra available at MassBank, acquired in $\mathrm{ESI}^{-}$at $20 \mathrm{eV}$ collision energy (Lege and Zwiener, 2015). This artificial sweetener has been previously reported in surface water 
Table 1

Compounds detected by UHPLC-IMS-HRMS target screening. Ionisation mode has been included, as well as the number of identifications in the river samples $(n=40)$ and the confidence level (1 and 2). Metabolite compounds are shown in italics.

\begin{tabular}{|c|c|c|c|c|}
\hline \multirow{2}{*}{ Compounds } & \multirow{2}{*}{ Use } & \multirow{2}{*}{$\begin{array}{l}\text { Ionisation } \\
\text { mode }\end{array}$} & \multicolumn{2}{|c|}{$\begin{array}{l}\text { Number of identifications (\% of } \\
\text { samples) }\end{array}$} \\
\hline & & & Level 1 & Level 2 \\
\hline \multicolumn{5}{|c|}{ Drugs of abuse and metabolites } \\
\hline Cocaine & Stimulant & $\mathrm{ESI}^{+}$ & $17(43)$ & $11(30)$ \\
\hline Benzoylecgonine & Stimulant & $\mathrm{ESI}^{+}$ & $19(48)$ & $6(15)$ \\
\hline \multicolumn{5}{|c|}{ Pharmaceuticals and metabolites } \\
\hline $4-A A$ & Analgesic & $\mathrm{ESI}^{+}$ & $1(3)$ & $11(28)$ \\
\hline $4-A A A$ & Analgesic & $\mathrm{ESI}^{+}$ & $15(38)$ & $4(10)$ \\
\hline $4-F A A$ & Analgesic & $\mathrm{ESI}^{+}$ & $13(33)$ & $8(20)$ \\
\hline \multirow[t]{2}{*}{ Acetaminophen ${ }^{1}$} & Analgesic & $\mathrm{ESI}^{+}$ & - & $14(35)$ \\
\hline & & $\mathrm{ESI}^{-}$ & - & $14(35)$ \\
\hline Atorvastatin & Cholesterol & $\mathrm{ESI}^{-}$ & $1(3)$ & - \\
\hline Carbamazepine & Anticonvulsant & $\mathrm{ESI}^{+}$ & $18(45)$ & - \\
\hline Clarithromycin & Antibiotic & $\mathrm{ESI}^{+}$ & - & $1(3)$ \\
\hline Clopidogrel carboxylic acid & Antiplatelet & $\mathrm{ESI}^{+}$ & $2(5)$ & $3(8)$ \\
\hline Codeine & Analgesic & $\mathrm{ESI}^{+}$ & - & $7(18)$ \\
\hline Diclofenac & Analgesic & $\mathrm{ESI}^{+}$ & $12(30)$ & - \\
\hline Iopamidol & Contrast agent & $\mathrm{ESI}^{-}$ & - & $2(5)$ \\
\hline Irbesartan & Hypertensive & $\mathrm{ESI}^{+}$ & $5(13)$ & - \\
\hline Ketoprofen & Analgesic & $\mathrm{ESI}^{+}$ & $5(13)$ & $5(13)$ \\
\hline Levamisole & Anthelmintic & $\mathrm{ESI}^{+}$ & $9(23)$ & $5(13)$ \\
\hline Lidocaine & Anesthetic & $\mathrm{ESI}^{+}$ & - & $11(28)$ \\
\hline Lincomycin & Antibiotic & $\mathrm{ESI}^{+}$ & $1(3)$ & - \\
\hline \multirow[t]{2}{*}{ Losartan } & Hypertensive & $\mathrm{ESI}^{+}$ & $17(43)$ & - \\
\hline & & ESI- & $17(43)$ & $1(3)$ \\
\hline \multirow[t]{2}{*}{ Losartan carboxylic acid } & Hypertensive & $\mathrm{ESI}^{+}$ & $16(40)$ & - \\
\hline & & $\mathrm{ESI}^{-}$ & $17(43)$ & - \\
\hline Mefenamic acid & Analgesic & $\mathrm{ESI}^{+}$ & $13(33)$ & - \\
\hline Metoprolol & Hypertensive & $\mathrm{ESI}^{+}$ & - & $12(30)$ \\
\hline Naproxen & Analgesic & $\mathrm{ESI}^{+}$ & $11(28)$ & $3(8)$ \\
\hline Oxacillin & Antibiotic & $\mathrm{ESI}^{+}$ & - & $1(3)$ \\
\hline Oxycodone & Analgesic & $\mathrm{ESI}^{+}$ & - & $1(3)$ \\
\hline Phenazone & Analgesic & $\mathrm{ESI}^{+}$ & $1(3)$ & $16(40)$ \\
\hline Sulfamethoxazole & Antibiotic & $\mathrm{ESI}^{+}$ & $1(3)$ & $15(38)$ \\
\hline \multirow[t]{2}{*}{ Telmisartan } & Hypertensive & $\mathrm{ESI}^{+}$ & $3(8)$ & - \\
\hline & & $\mathrm{ESI}^{-}$ & $1(3)$ & $1(3)$ \\
\hline Trimethoprim & Antibiotic & $\mathrm{ESI}^{+}$ & $13(33)$ & - \\
\hline \multirow[t]{2}{*}{ Valsartan $^{1}$} & Hypertensive & $\mathrm{ESI}^{+}$ & $13(33)$ & $3(8)$ \\
\hline & & $\mathrm{ESI}^{-}$ & $15(38)$ & $3(8)$ \\
\hline Venlafaxine & Antidepressant & $\mathrm{ESI}^{+}$ & - & $1(3)$ \\
\hline Venlafaxine 0 -desmethyl & Antidepressant & $\mathrm{ESI}^{+}$ & - & $12(30)$ \\
\hline \multicolumn{5}{|l|}{ Other compounds } \\
\hline \multirow[t]{2}{*}{ Sucralose $\mathrm{e}^{1,2}$} & Sweetener & $\mathrm{ESI}^{+}$ & - & $1(3)$ \\
\hline & & $\mathrm{ESI}^{-}$ & - & $19(48)$ \\
\hline Methylparaben & Preservative & $\mathrm{ESI}^{-}$ & - & $7(18)$ \\
\hline Propylparaben & Preservative & $\mathrm{ESI}^{-}$ & $3(8)$ & $13(33)$ \\
\hline Benzophenone-3 & UV filter & $\mathrm{ESI}^{+}$ & $12(30)$ & $6(15)$ \\
\hline
\end{tabular}

Compound identified at Level 2 of confidence, but detected in $\mathrm{ESI}^{+}$and $\mathrm{ESI}^{-}$.

No fragment ions were observed. The identification was performed by accurate mass and the presence of the $\mathrm{Cl}_{3}$ isotope pattern.

samples (urban estuary) by LC-HRMS (ESI ${ }^{-}$) based on the presence of the deprotonated molecule and the fragment ion corresponding to ${ }^{37} \mathrm{Cl}^{-}$ion $(\mathrm{m} / \mathrm{z} 36.9686)$ (Tian et al., 2020). In our work, HRMS operated in the $m / z$ 50-1000 mass range (typically used in HRMS wide-scope screening strategies), and therefore the fragment ion corresponding to chlorine was not acquired and consequently could not be observed. In addition, sucralose presented in most cases a RT deviation higher than 0.1 min when compared to its analytical standard. RT shifts have been associated to analyte interaction with matrix interferences during chromatographic separation (Celma et al., 2020), reducing the confidence on compound identification. At this point, it is worth noticing the usefulness of IMS, as it provides a matrix-independent parameter for compound identification. The fact that CCS values are not affected, even in complexmatrix samples, gives higher confidence to the identification process in HRMS-based screening strategies, especially in cases such as sucralose detected in Amazon water samples. As shown in this work, CCS together with the isotope pattern and accurate mass can solve identification of compounds in cases where the RT does not fit with the reference standard.

Even when standards were available, some compounds were only identified at Level 2, as no fragment ions were detected (probably due to the low concentration levels in samples or due to variations in the RT), as previously explained. In the case of the contrast medium compound iopamidol, detected in $\mathrm{ESI}^{-}$, no fragment ions were observed in two samples. This limited our capacity to differentiate between iopamidol and iomeprol (another contrast medium), as both compounds are structurally similar. In addition, CCS values for both compounds are rather similar $\left(205.65 \AA^{2}\right.$ for iopamidol and $211.20 \AA^{2}$ for iomeprol, for the deprotonated molecules) Thus, CCS deviations calculated for both compounds were below $2 \%$ (experimental CCS $207.54 \AA^{2}$, CCS deviation $0.92 \%$ for iopamidol and $-1.73 \%$ for iomeprol), making identification troublesome. In this case, the compound could be identified as iopamidol based on RT, as the suspect compound had a RT deviation of $0.07 \mathrm{~min}$, while for iomeprol was $-0.40 \mathrm{~min}$. Therefore, despite the 


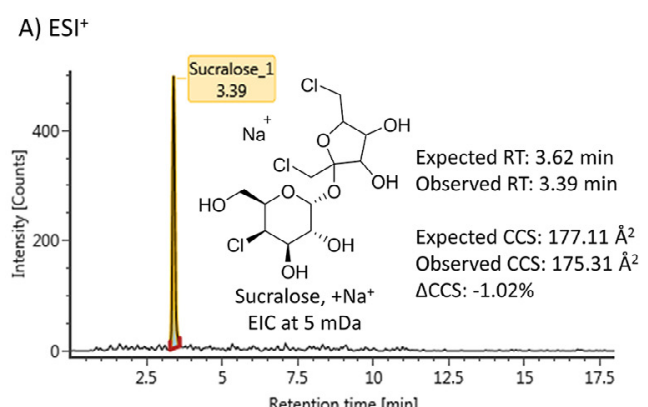

B) $\mathrm{ESI}^{-}$

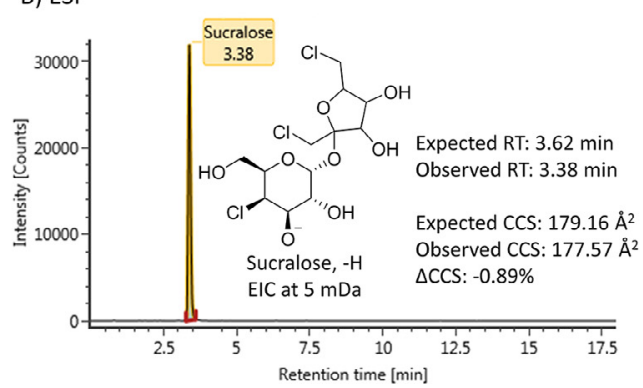

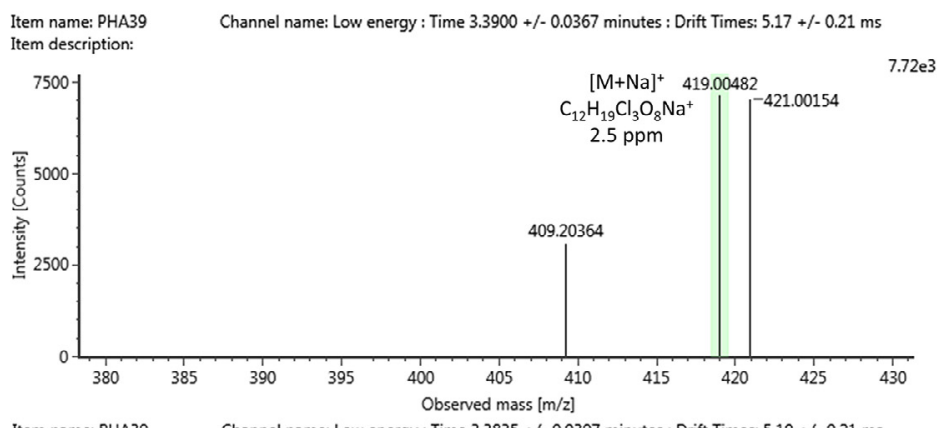

Item name: PHA39 Channel name: Low energy : Time $3.3835+/-0.0397$ minutes : Drift Times: $5.10+/-0.21 \mathrm{~ms}$ Item description:

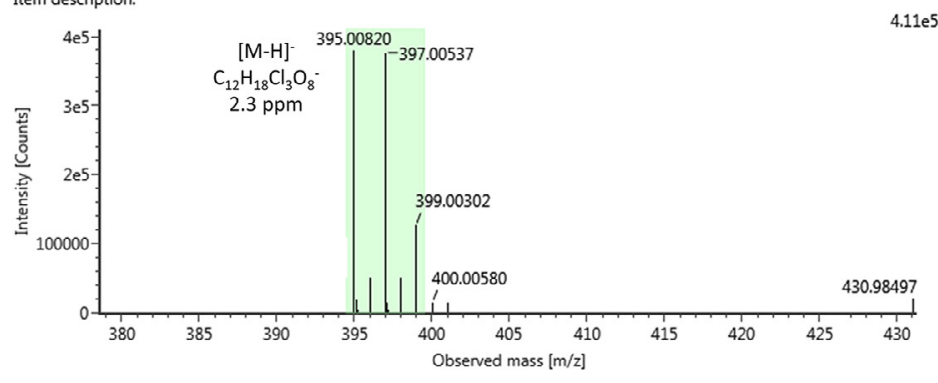

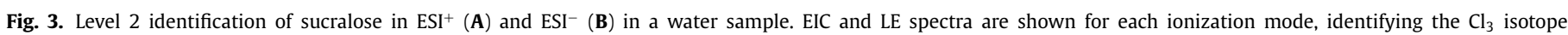
pattern.

great potential of IMS in HRMS methodologies, LC data provided pivotal information for the identification of isomeric compounds. Therefore, the power of LC-IMS-HRMS comes from the combination of the different useful information provided: chromatographic RT, CCS values and accurate-mass data.

\subsection{Suspect screening results}

For suspect screening, an in-house database containing 631 compounds was used, including parent compounds and metabolites and transformation products previously found in water samples. As stated in the Section 2.5.2, a three-step workflow was developed to simplify data evaluation and minimize the number of false positives, including the CCS predicted as an important parameter related to ion mobility, and applying different confidence levels to the tentative identifications (following Celma et al., 2020).

A total of 15 compounds were tentatively identified at different confidence levels in the suspect screening. Table 2 shows the list of compounds identified, indicating the ionization mode, the confidence level, and the number of identifications. As illustrative example, Fig. 4 shows the identification of the antiretroviral atazanavir (1.7 ppm mass error) at Level 2. LE and HE spectra $\left(\mathrm{ESI}^{+}\right)$are shown without $(\mathbf{A})$ and with $(\mathbf{B})$ drift alignment. After drift alignment, only those fragment ions with the same drift time than the protonated molecule remained. The experimental CCS had a $-1.85 \%$ deviation with respect to the predicted one, and up to 4 accurate mass fragments (below -3.6 ppm mass error) were justified based on compound structure (Fig. 4B). The lack of reference standard made the identification at Level 1 unfeasible. However, there was relevant and abundant information to consider its identification as reliable.

Several identifications were made at the confidence Level 3. This level included compounds with plausible accurate-mass fragments and CCS deviations $\leq 6 \%$ of the predicted values. The Level 3 also included compounds which RT, CCS, fragmentation data and isotope pattern might be compatible with different structures. This was the case of the hydroxylated metabolite of diclofenac (Table 2, "Diclofenac, (3,4,5)-hydroxy"), as the exact position of the hydroxyl group in one of the aromatic rings could not be stablished based on the observed fragmentation. Fig. 5 shows the drift-aligned LE/HE spectra for the hydroxylated metabolite of the only chromatographic peak observed (Fig. 5A), as well as the chromatogram and drift-aligned LE/HE spectra (Fig. 5B) for parent diclofenac, both compounds detected in the same water sample. The experimental CCS $\left(161.01 \AA^{2}\right)$ was below $2 \%$ deviation with respect to all predicted CCS values for the different positions of the hydroxyl group (3-hydroxy $159.94 \AA^{2}$, 4-hydroxy $160.08 \AA^{2}$, 5hydroxy $161.39 \AA^{2}$ ). Under these conditions, it was unfeasible to identify the right isomer present in the sample. The hydroxyl moiety can be located in one of the aromatic rings, as the fragment ion observed for this metabolite (Fig. 5A, right) presents a $16 \mathrm{Da}$ shift respect to the diclofenac fragment ion (Fig. 5B, right). Moreover, the isotope pattern corresponding to two chlorine atoms was observed for both protonated molecules (parent and metabolite) as well as the isotope pattern of one chlorine for fragment ions, supporting the metabolite identification. Nevertheless, only Level 3 of confidence could be reached, as the exact position of the hydroxyl could not be unequivocally stablished.

At identification Level 4, only the (de)protonated molecule was detected (no fragment ions were observed), but the predicted CCS presented a deviation $\leq 6 \%$. As shown in Table 2 , norcocaethylene, atazanavir, phenytoin and cotinine were identified at Level 4 in some samples, but at Level 3 and 2 in others. Detections at Level 4 presented the same RT than positives identified at Level 3 and 2 , therefore increasing the confidence on the compound identification despite the absence of fragment ions. This was surely related to the low analyte concentrations in certain samples.

\subsection{Contamination patterns in the Amazon River}

Through this study we have identified 51 compounds at different confidence levels, 36 in target screening (Table 1) and 15 in the suspect screening (Table 2), belonging to 18 different chemical use categories. Samples taken in the urban streams show a significantly higher number of compounds as compared to those taken in the Amazon River and its main tributaries, particularly in Manaus, Belém and Macapá, with compound mixtures formed by 
Table 2

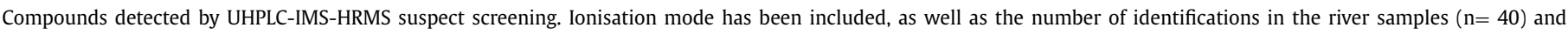
the confidence level (2-4). Metabolite compounds are shown in italics.

\begin{tabular}{|c|c|c|c|c|c|}
\hline \multirow{2}{*}{ Compounds } & \multirow{2}{*}{ Use } & \multirow{2}{*}{$\begin{array}{l}\text { Ionisation } \\
\text { mode }\end{array}$} & \multicolumn{3}{|c|}{ Number of identifications (\% of samples) } \\
\hline & & & Level 2 & Level 3 & Level 4 \\
\hline \multicolumn{6}{|c|}{ Drugs of abuse and metabolites } \\
\hline Norcocaethylene & Stimulant & $\mathrm{ESI}^{+}$ & $4(10)$ & $4(10)$ & $3(8)$ \\
\hline \multicolumn{6}{|c|}{ Pharmaceuticals and metabolites } \\
\hline Atazanavir & Antiretroviral & $\mathrm{ESI}^{+}$ & $10(25)$ & $3(8)$ & $1(3)$ \\
\hline Atorvastatin, 4-hydroxy & Cholesterol & $\mathrm{ESI}^{-}$ & - & $3(8)$ & - \\
\hline Codeine & Analgesic & $\mathrm{ESI}^{+}$ & - & - & $2(5)$ \\
\hline Diclofenac, $(3,4,5)$-hydroxy ${ }^{1}$ & Analgesic & $\mathrm{ESI}^{+}$ & - & $14(35)$ & - \\
\hline Fluconazole & Antifungal & $\mathrm{ESI}^{+}$ & $16(40)$ & - & - \\
\hline Hydrochlorothiazide & Diuretic/Hypertensive & $\mathrm{ESI}^{-}$ & $9(23)$ & $6(15)$ & - \\
\hline Losartan carboxaldehyde & Hypertensive & $\mathrm{ESI}^{+}$ & $13(33)$ & - & - \\
\hline Meclofenamic acid & Analgesic & $\mathrm{ESI}^{+}$ & $13(33)$ & $3(8)$ & - \\
\hline Metoprolol acid & Hypertensive & $\mathrm{ESI}^{+}$ & $9(23)$ & $4(10)$ & - \\
\hline Phenytoin & Anticonvulsant & $\mathrm{ESI}^{+}$ & - & $14(35)$ & $2(5)$ \\
\hline Sulfociprofloxacin & Antibiotic & $\mathrm{ESI}^{-}$ & $7(18)$ & $5(13)$ & - \\
\hline \multicolumn{6}{|l|}{ Other compounds } \\
\hline Caffeine & Stimulant & $\mathrm{ESI}^{+}$ & $23(58)$ & $17(43)$ & - \\
\hline Paraxanthine & Stimulant & $\mathrm{ESI}^{+}$ & $14(35)$ & $4(10)$ & - \\
\hline Cotinine & Alkaloid & $\mathrm{ESI}^{+}$ & - & $7(18)$ & $16(40)$ \\
\hline
\end{tabular}

1 The exact position of the hydroxyl group in the chlorinated aromatic ring could not be stablished.

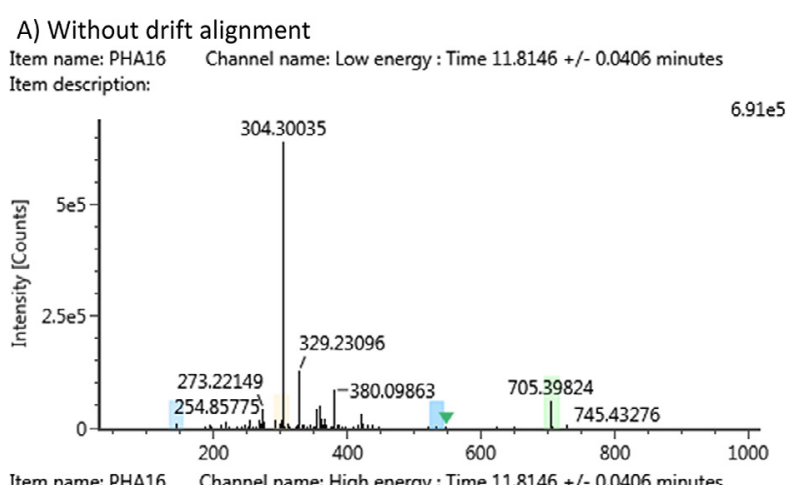

B) With drift alignment

Item name: PHA16 Channel name: Low energy : Time $11.8146+/-0.0406$ minutes :91e5 Item description:
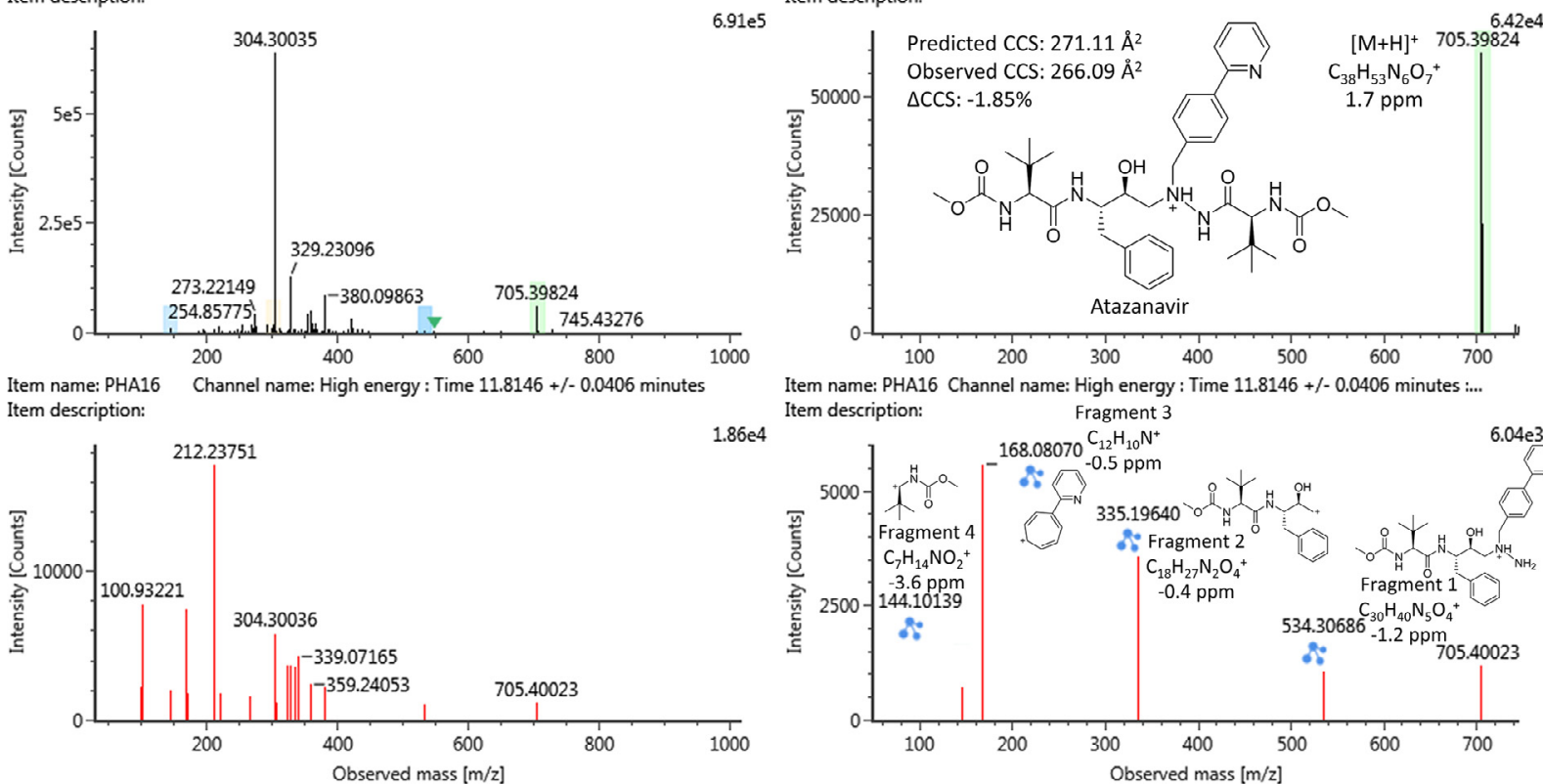

$$
\text { Item description: Fragment } 3
$$

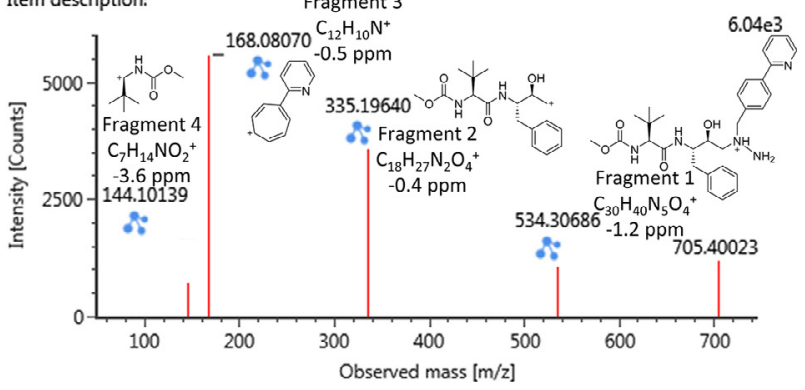

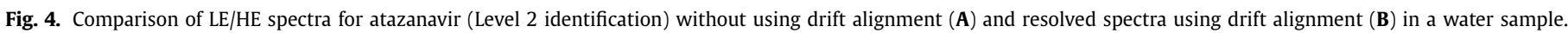
Fragment ions are identified in HE resolved spectrum.

up to 35-40 different substances, belonging to 15 different compound categories (Fig. 6). These samples contain a relatively similar composition, regarding the number and type of substances identified, except for sample B2 (taken in Belém), which was taken in an area with notable dilution by the Tocantins River and affected by marsh tides. Our results also indicate that the samples taken in Santarem show a lower number of compounds (8-11) as compared to those taken in the other urban areas. This can be explained by the smaller population of this city, which represents approximately $15 \%$ of the population of Manaus and Belém, and the higher dilution potential (Fig. 6).

We observed that the predominant substance groups (in terms of number of compounds) in the samples taken in areas with significant urban impact were analgesics and anti-hypertensives, followed by stimulants and antibiotics (Fig. 6). The most frequently detected analgesics were acetaminophen, phenazone, mefenamic and meclofenamic acids, diclofenac, naproxen, codeine, ketoprofen, as well as the human metabolites of metamizole (4-AA, 4-AAA, 4-FAA). The group of anti-hypertensives was dominated by valsartan, losartan, metoprolol and their metabolites. Regarding stimulants, the compounds with the highest detection rates in samples with urban impact were caffeine and its metabolite (paraxanthine), and cocaine and its metabolite (benzoylecgonine). As for antibiotics, the highest detection rate was found for sulfamethoxazole, trimethoprim, and the metabolite of ciprofloxacin (sulfociprofloxacin). Other compounds that showed high frequency of detection ( $\geq 30 \%$ of samples) were the anticonvulsant carbamazepine, the UV filter benzophenone-3, the antifungal fluconazole, and the metabolites of nicotine (cotinine) and the antidepressant venlafaxine (venlafaxine 0 -desmethyl). For a detailed 

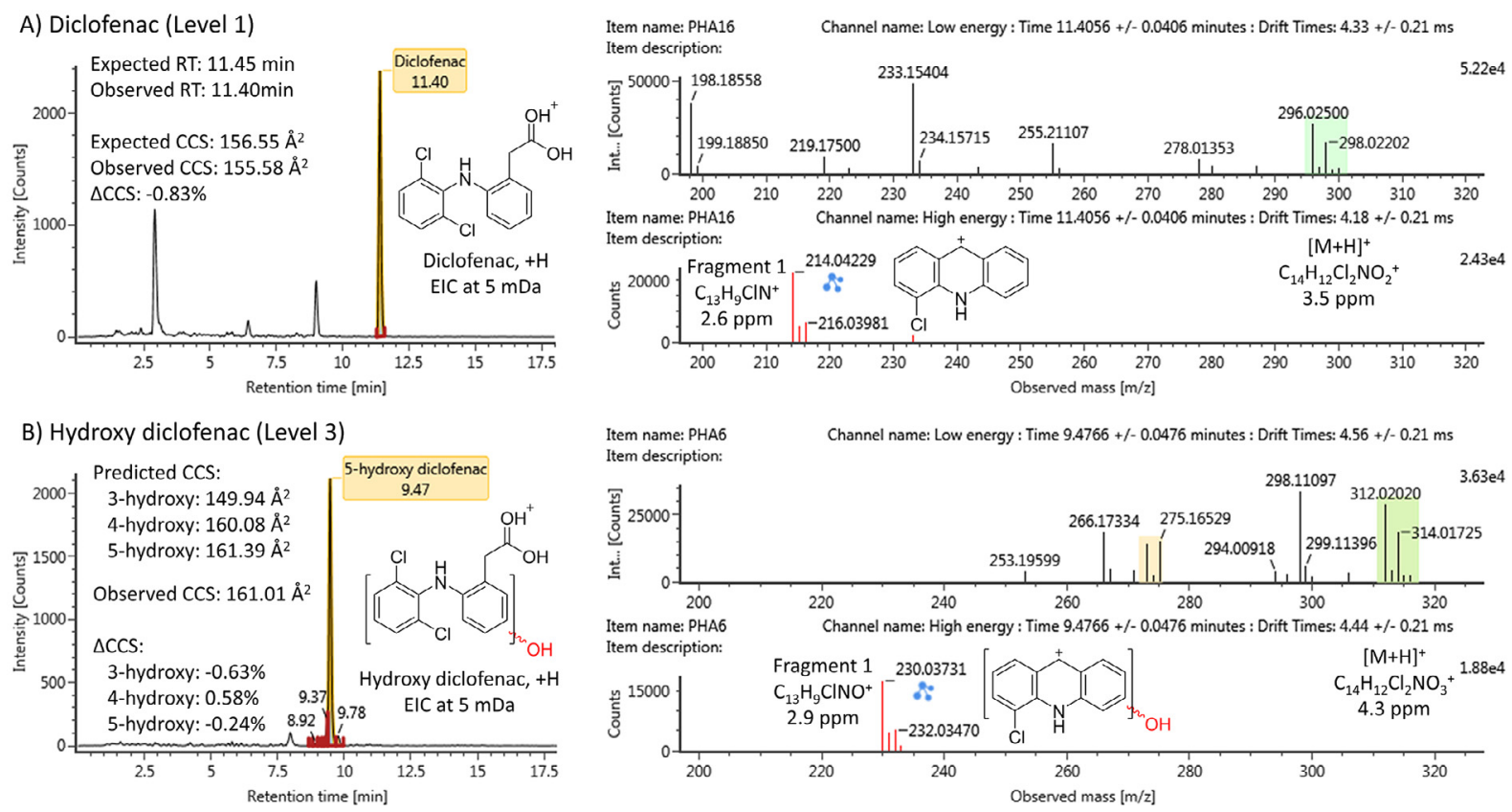

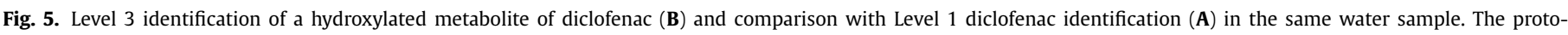
nated molecule and fragment ion for both compounds are highlighted, illustrating the 16 Da shift corresponding to a hydroxylation.

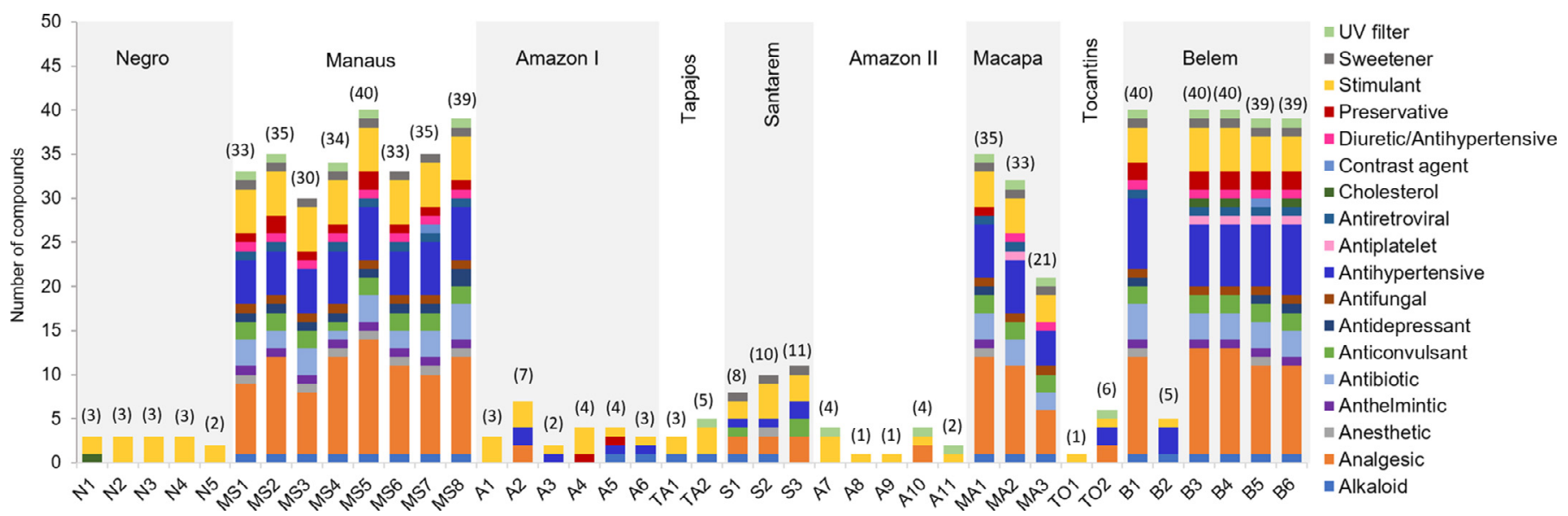

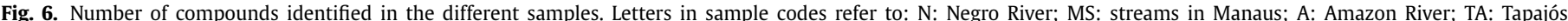
River; S: streams in Santarém; MA: streams in Macapá; TO: Tocantins River; B: streams in Belém.

description of the compounds found in each sample, see Tables S2 and $\mathbf{S 3}$ in the Supplementary Information.

The results of the screening performed in urban streams does not show marked discrepancies with other wide-scope screening exercises performed in rivers of Europe (Fonseca et al., 2020; Rico et al., 2019), suggesting that the consumption patterns of the major pharmaceutical groups and other drugs may be rather similar. For instance, Rico et al. (2019) found that caffeine, nicotine, valsartan, carbamazepine, acetaminophen, diclofenac, ketoprofen, sulfamethoxazole and trimethoprim, were amongst the most frequently detected substances in rivers with significant anthropogenic impact of Central Spain. The results of quantitative analyses and preliminary risk assessments performed in other countries of Latin America point at compounds such as carbamazepine, acetaminophen, sulfamethoxazole and trimethoprim as posing potential hazards for freshwater ecosystems (Valdez-Carrillo et al., 2020). Furthermore, the statistical comparisons made by these authors show no differences between exposure patterns of treated and untreated wastewaters, pointing to a need to improve the san- itation system and the treatment technologies in Brazil and other regions of South America (Valdez-Carrillo et al., 2020). Given to the fact that most of the wastewaters emitted within the sampled urban areas are discharged untreated into the Amazon river or its tributaries, it is of utmost importance to quantify exposure levels and conduct chemical prioritization studies to determine substances with potential contribution to freshwater biodiversity loss near urban areas.

Our study shows that the number of compounds identified in the Amazon River and its main tributaries was significantly lower as compared to the urban streams (Fig. 6), indicating a notable dilution potential of the contaminated wastewaters and/or dissipation of the test substances. In this way, samples taken in the dilution area of Manaus (N4 and N5) only contained a limited number of compounds (2-3). The highest number of compounds was detected in samples A2 (7 compounds) and TO2 (6 compounds), which correspond to the dilution area of the city of Iranduba (ca. 50,000 inhabitants) and Belém, respectively. The compounds with the highest prevalence in the Amazon River and its tributaries 
were caffeine, cocaine and its metabolite benzoylecgonine, which were detected in a large number of samples, including those taken in the Anavilhanas National Park (N1 and N2).

The detection of benzoylecgonine in the water cycle has been reported in the literature (Thomas et al., 2014; van der Aa et al., 2013, Hernandez et al., 2015a), including urban wastewater, which allowed the estimation of cocaine consumption rates in the population of specific geographical regions or cities (Bijlsma et al., 2016; Hernández et al., 2015a; Maldaner et al., 2012). Most studies dealing with the investigation of illicit drugs in environmental and wastewater samples report the presence of benzoylecgonine, and less frequently its parent compound (cocaine), revealing cocaine consumption in the area under study. However, in the present work cocaine was detected in $73 \%$ of the samples, while benzoylecgonine was in $63 \%$. The higher frequency of detection of the unaltered drug might be related to the production or processing laboratories of cocaine in various areas of the Amazon (Dávalos et al., 2016), and subsequent discharges of the drug into the river. In this regard, Thomas et al. (2014) found cocaine and benzoylecgonine in most samples taken from two streams crossing the urban area of Manaus with concentrations up to 5.9 and $3.6 \mu \mathrm{g} / \mathrm{L}$, respectively, and found higher concentrations of cocaine as compared to those of its metabolite in several sampling sites.

The antidepressant venlafaxine and its main urinary metabolite, $O$-desmethyl venlafaxine, were also detected in several samples, mainly the metabolite (found in 12 samples). The presence of this pharmaceutical has been reported in environmental water samples in other parts of the world (Fonseca et al., 2020; Hernández et al., 2014; Kern et al., 2009). Both compounds, venlafaxine and its $O$ desmethyl metabolite, as well as some of the antibiotics detected here (sulfamethoxazole, trimethoprim) have been recently included in the Watch List of substances that should be controlled in Europe (European Commission, 2020), illustrating the concern about the presence of these compounds in the aquatic environment and the need to have more detailed information on their exposure and risks. Furthermore, the detection of several metabolites in Amazonian waters (e.g. O-desmethyl venlafaxine, losartan carboxylic acid, losartan carboxaldehyde, hydroxy diclofenac, 4-hydroxy atorvastatin, sulfociprofloxacin) supports the need to include them in further monitoring and risk assessment studies, particularly since they might be biologically active and exert long-term toxicity to a wide range of living organisms (Pereira et al., 2020a, 2020b).

\section{Conclusions}

This study provides the first wide-scope monitoring of pharmaceuticals, illicit drugs, and metabolites in different areas of the Amazon basin. Here we show that the combination of target and suspect screening, based on LC-IMS-HRMS, is a powerful tool for assessing the presence of these substances in samples with an ample gradient of anthropogenic pressure. Data provided by IMS-HRMS have increased the confidence on compound identification by the use of CCS as identification parameter, as well as the fragment ions drift alignment with the corresponding ionized molecule, which is of particular relevance in tentative identifications (i.e., suspect screening). The results of this study showed relevant presence of pharmaceuticals in the Amazon River and tributaries, with 51 different compounds and metabolites being identified. This study demonstrates that analgesics and antihypertensive drugs are commonly found in areas with significant urban impact, and some stimulants such as caffeine or illicit drug (cocaine) residues may also be found in relatively remote areas. The suspect screening also allowed the identification of metabolites, some of which should also be included in further ecotoxicological evaluations. Finally, our study supports the need to improve the sanitation system of urban areas in the Amazon to re- duce chemical emissions, and recommends performing follow-up studies to quantify chemical exposure levels and to assess risks for Amazonian freshwater ecosystems.

\section{Supplementary Information.pdf}

Wide-scope screening of pharmaceuticals, illicit drugs and their metabolites in the Amazon River

\section{Declaration of Competing Interest}

The authors declare that they do not have competing financial interests or personal relationships that could have appeared to influence the work reported in this paper.

\section{Acknowledgements}

This study has been funded by the National Geographic Society through an early career grant to A. Rico (EC-59809C-19), the Universitat Jaume I (UJI) (Projects B2018-19 and B2018-55), the Spanish Ministry of Science, Innovation and Universities (RTI2018097417-B-100) and the Generalitat Valenciana (Research Group of Excellence Prometeo 2019/040). A. Rico acknowledges the Ramón y Cajal grant provided by the Spanish Ministry of Science and Innovation (RYC2019-028132-I). D. Fabregat-Safont acknowledges Ministerio de Educación, Cultura y Deporte in Spain for his predoctoral grant (FPU15/02033). R. Oliveira acknowledges "Fundação de Amparo à Pesquisa do Estado de São Paulo" - FAPESP (project 2018/03108-0). We thank all citizens, boatmen and technicians that helped during the field sampling campaign.

\section{Supplementary materials}

Supplementary material associated with this article can be found, in the online version, at doi:10.1016/j.watres.2021.117251.

\section{References}

Bade, R., Bijlsma, L., Miller, T.H., Barron, L.P., Sancho, J.V., Hernández, F., 2015. Suspect screening of large numbers of emerging contaminants in environmental waters using artificial neural networks for chromatographic retention time prediction and high resolution mass spectrometry data analysis. Sci. Total Environ. 538, 934-941. doi:10.1016/j.scitotenv.2015.08.078.

Begossi, A., Salivonchyk, S.V., Hallwass, G., Hanazaki, N., Lopes, P.F.M., Silvano, R.A.M., Dumaresq, D., Pittock, J., 2019. Fish consumption on the Amazon: a review of biodiversity, hydropower and food security issues. Brazilian J. Biol. 79, 345-357. doi:10.1590/1519-6984.186572.

Bijlsma, L., Bade, R., Celma, A., Mullin, L., Cleland, G., Stead, S., Hernandez, F., Sancho, J.V., 2017. Prediction of collision cross-section values for small molecules: application to pesticide residue analysis. Anal. Chem. 89, 6583-6589. doi:10. 1021/acs.analchem.7b00741.

Bijlsma, L., Berntssen, M.H.G., Merel, S., 2019. A refined nontarget workflow for the investigation of metabolites through the prioritization by in silico prediction tools. Anal. Chem. 91, 6321-6328. doi:10.1021/acs.analchem.9b01218.

Bijlsma, L., Botero-Coy, A.M., Rincón, R.J., Peñuela, G.A., Hernández, F., 2016. Estimation of illicit drug use in the main cities of Colombia by means of urban wastewater analysis. Sci. Total Environ. 565, 984-993. doi:10.1016/j.scitotenv. 2016.05.078

Boix, C., Ibáñez, M., Bagnati, R., Zuccato, E., Sancho, J.V., Hernández, F., Castiglioni, S., 2016a. High resolution mass spectrometry to investigate omeprazole and venlafaxine metabolites in wastewater. J. Hazard. Mater. 302, 332-340. doi:10.1016/ j.jhazmat.2015.09.059.

Boix, C., Ibáñez, M., Sancho, J.V., Parsons, J.R., Voogt, P.de, Hernández, F., 2016b. Biotransformation of pharmaceuticals in surface water and during waste water treatment: identification and occurrence of transformation products. J. Hazard. Mater. 302, 175-187. doi:10.1016/j.jhazmat.2015.09.053.

Boix, C., Ibáñez, M., Sancho, J.V., Rambla, J., Aranda, J.L., Ballester, S., Hernández, F., 2014. Fast determination of 40 drugs in water using large volume direct injection liquid chromatography-tandem mass spectrometry. Talanta 131, 719-727. doi:10.1016/j.talanta.2014.08.005.

Canellas, E., Vera, P., Nerín, C., 2019. Ion mobility quadrupole time-of-flight mass spectrometry for the identification of non-intentionally added substances in UV varnishes applied on food contact materials. A safety by design study. Talanta 205, 120103. doi:10.1016/j.talanta.2019.06.103. 
Canlı, O., Çetintürk, K., Öktem Olgun, E.E., 2020. Determination of 117 endocrine disruptors (EDCs) in water using SBSE TD-GC-MS/MS under the European water framework directive. Anal. Bioanal. Chem. 412, 5169-5178. doi:10.1007/ s00216-020-02553-4.

Castello, L., Macedo, M.N., 2016. Large-scale degradation of Amazonian freshwater ecosystems. Glob. Chang. Biol. 22, 990-1007. doi:10.1111/gcb.13173.

Celma, A., Sancho, J.V., Schymanski, E.L., Fabregat-Safont, D., Ibáñez, M., Goshawk, J., Barknowitz, G., Hernández, F., Bijlsma, L., 2020. Improving target and suspect screening high-resolution mass spectrometry workflows in environmental analysis by ion mobility separation. Environ. Sci. Technol. 54, 15120-15131. doi:10.1021/acs.est.0c05713.

Chaves, M.de J.S., Barbosa, S.C., Malinowski, M.de M., Volpato, D., Castro, Í.B., Franco, T.C.R.dos S., Primel, E.G., 2020. Pharmaceuticals and personal care products in a Brazilian wetland of international importance: Occurrence and environmental risk assessment. Sci. Total Environ. 734, 139374. doi:10.1016/j. scitotenv.2020.139374.

Choi, Y., Lee, J.-H., Kim, K., Mun, H., Park, N., Jeon, J., 2021. Identification, quantification, and prioritization of new emerging pollutants in domestic and industrial effluents, Korea: Application of LC-HRMS based suspect and nontarget screening. J. Hazard. Mater. 402, 123706. doi:10.1016/j.jhazmat.2020. 123706.

Dávalos, L.M., Sanchez, K.M., Armenteras, D., 2016. Deforestation and coca cultivation rooted in twentieth-century development projects. Bioscience 66, 974-982. doi:10.1093/biosci/biw118.

European Commission, 2020. Decision (EU) 2020/1161 of 4 August 2020 establishing a watch list of substances for Union-wide monitoring in the field of water policy pursuant to Directive 2008/105/EC of the European Parliament and of the Council. Off. J. Eur. Union L 257, 32-35.

Ferreira, M.da S., Fontes, M.P.F., Pacheco, A.A., Ker, J.C., Lima, H.N., 2021. Health risks of potentially toxic trace elements in urban soils of Manaus city, Amazon, Brazil. Environ. Geochem. Health 0. doi:10.1007/s10653-021-00834-0.

Fonseca, E., Hernández, F., Ibáñez, M., Rico, A., Pitarch, E., Bijlsma, L., 2020. Occurrence and ecological risks of pharmaceuticals in a Mediterranean river in Eastern Spain. Environ. Int. 144, 106004. doi:10.1016/j.envint.2020.106004

Fonseca, E., Renau-Pruñonosa, A., Ibáñez, M., Gracia-Lor, E., Estrela, T., Jiménez, S., Pérez-Martín, M.Á., González, F., Hernández, F., Morell, I., 2019. Investigation of pesticides and their transformation products in the Júcar River Hydrographical Basin (Spain) by wide-scope high-resolution mass spectrometry screening. Environ. Res. 177, 108570. doi:10.1016/j.envres.2019.108570.

Gracia-Lor, E., Sancho, J.V., Hernández, F., 2011. Multi-class determination of around 50 pharmaceuticals, including 26 antibiotics, in environmental and wastewater samples by ultra-high performance liquid chromatography-tandem mass spectrometry. J. Chromatogr. A 1218, 2264-2275. doi:10.1016/j.chroma.2011. 02.026 .

Guardian, M.G.E., Antle, J.P., Vexelman, P.A., Aga, D.S., Simpson, S.M., 2021. Resolving unknown isomers of emerging per- and polyfluoroalkyl substances (PFASs) in environmental samples using COSMO-RS-derived retention factor and mass fragmentation patterns. J. Hazard. Mater. 402, 123478. doi:10.1016/j.jhazmat. 2020.123478

Hernández, F., Ibáñez, M., Bade, R., Bijlsma, L., Sancho, J.V., 2014. Investigation of pharmaceuticals and illicit drugs in waters by liquid chromatography-highresolution mass spectrometry. TrAC Trends Anal. Chem. 63, 140-157. doi:10. 1016/j.trac.2014.08.003

Hernández, F., Ibáñez, M., Botero-Coy, A.-M., Bade, R., Bustos-López, M.C., Rincón, J., Moncayo, A., Bijlsma, L., 2015a. LC-QTOF MS screening of more than 1,000 licit and illicit drugs and their metabolites in wastewater and surface waters from the area of Bogotá, Colombia. Anal. Bioanal. Chem. 407, 6405-6416. doi:10.1007/ s00216-015-8796-X.

Hernández, F., Ibáñez, M., Portolés, T., Cervera, M.I., Sancho, J.V., López, F.J., 2015b. Advancing towards universal screening for organic pollutants in waters. J. Hazard. Mater. 282, 86-95. doi:10.1016/j.jhazmat.2014.08.006.

Hinnenkamp, V., Balsaa, P., Schmidt, T.C., 2019. Quantitative screening and prioritization based on UPLC-IM-Q-TOF-MS as an alternative water sample monitoring strategy. Anal. Bioanal. Chem. 411, 6101-6110. doi:10.1007/s00216-01901994-w.

Hollender, J., Schymanski, E.L., Singer, H.P., Ferguson, P.L., 2017. Nontarget screening with high resolution mass spectrometry in the environment: ready to go? Environ. Sci. Technol. 51, 11505-11512. doi:10.1021/acs.est.7b02184

Hurd, L.E., Sousa, R.G.C., Siqueira-Souza, F.K., Cooper, G.J., Kahn, J.R., Freitas, C.E.C., 2016. Amazon floodplain fish communities: habitat connectivity and conservation in a rapidly deteriorating environment. Biol. Conserv. 195, 118-127. doi:10. 1016/j.biocon.2016.01.005

IBGE. Brazilian Institute of Geography and Statistics, 2020. Brazilian Cens [WWW Document]. URL https://www.ibge.gov.br/ (accessed 9.23.20).

Jézéquel, C., Tedesco, P.A., Darwall, W., Dias, M.S., Frederico, R.G., Hidalgo, M., Hugueny, B., Maldonado-Ocampo, J., Martens, K., Ortega, H., Torrente-Vilara, G., Zuanon, J., Oberdorff, T., 2020. Freshwater fish diversity hotspots for conservation priorities in the Amazon Basin. Conserv. Biol. 34, 956-965. doi:10.1111/cobi. 13466

Kern, S., Fenner, K., Singer, H.P., Schwarzenbach, R.P., Hollender, J., 2009. Identification of transformation products of organic contaminants in natural waters by computer-aided prediction and high-resolution mass spectrometry. Environ. Sci. Technol. 43, 7039-7046. doi:10.1021/es901979h.

Lege, S., Zwiener, C., 2015. Sucralose; LC-ESI-QTOF; MS2; CE: 20.0V; [M-H]- [WWW Document]. MassBank.eu. URL https://massbank.eu/MassBank/RecordDisplay? id=TUE00666\&dsn=Env_Anal_Chem_U_Tuebingen (accessed 12.15.20).
Llorca, M., Vega-Herrera, A., Schirinzi, G., Savva, K., Abad, E., Farré, M., 2021. Screening of suspected micro(nano)plastics in the Ebro Delta (Mediterranean Sea). J. Hazard. Mater. 404, 124022. doi:10.1016/j.jhazmat.2020.124022.

Lotfi Khatoonabadi, R., Vosough, M., Hohrenk, L.L., Schmidt, T.C., 2021. Employing complementary multivariate methods for a designed nontarget LC-HRMS screening of a wastewater-influenced river. Microchem. J. 160, 105641. doi:10. 1016/j.microc.2020.105641.

Maldaner, A.O., Schmidt, L.L., Locatelli, M.A.F., Jardim, W.F., Sodré, F.F., Almeida, F.V., Pereira, C.E.B., Silva, C.M., 2012. Estimating cocaine consumption in the Brazilian Federal District (FD) by sewage analysis. J. Braz. Chem. Soc. 23, 861-867. doi:10. 1590/S0103-50532012000500011.

Menger, F., Gago-Ferrero, P., Wiberg, K., Ahrens, L., 2020. Wide-scope screening of polar contaminants of concern in water: A critical review of liquid chromatography-high resolution mass spectrometry-based strategies. Trends Environ. Anal. Chem. 28, e00102. doi:10.1016/j.teac.2020.e00102.

Mullin, L., Jobst, K., DiLorenzo, R.A., Plumb, R., Reiner, E.J., Yeung, L.W.Y., Jogsten, I.E., 2020. Liquid chromatography-ion mobility-high resolution mass spectrometry for analysis of pollutants in indoor dust: Identification and predictive capabilities. Anal. Chim. Acta 1125, 29-40. doi:10.1016/j.aca.2020.05.052.

Oberdorff, T., Dias, M.S., Jézéquel, C., Albert, J.S., Arantes, C.C., Bigorne, R., CarvajalValleros, F.M., De Wever, A., Frederico, R.G., Hidalgo, M., Hugueny, B., Leprieur, F. Maldonado, M., Maldonado-Ocampo, J., Martens, K., Ortega, H., Sarmiento, J., Tedesco, P.A., Torrente-Vilara, G., Winemiller, K.O., Zuanon, J., 2019. Unexpected fish diversity gradients in the Amazon basin. Sci. Adv. 5, eaav8681. doi:10.1126/ sciadv.aav8681.

Pereira, A., Silva, L., Laranjeiro, C., Lino, C., Pena, A., 2020a. Selected pharmaceuticals in different aquatic compartments: part I-source, fate and occurrence. Molecules 25, 1026. doi:10.3390/molecules25051026.

Pereira, A., Silva, L., Laranjeiro, C., Lino, C., Pena, A., 2020b. Selected pharmaceuticals in different aquatic compartments: Part II-toxicity and environmental risk assessment. Molecules 25, 1796. doi:10.3390/molecules25081796.

Pérez, S., Barceló, D., 2007. Application of advanced MS techniques to analysis and identification of human and microbial metabolites of pharmaceuticals in the aquatic environment. TrAC - Trends Anal. Chem. 26, 494-514. doi:10.1016/j.trac. 2007.05.004.

Oliveira, Puppim de, J.A., Balaban, O., Doll, C.N.H., Moreno-Peñaranda, R., Gasparatos, A., Iossifova, D., Suwa, A., 2011. Cities and biodiversity: Perspectives and governance challenges for implementing the convention on biological diversity (CBD) at the city level. Biol. Conserv. 144, 1302-1313. doi:10.1016/j.biocon.2010.12.007.

Rico, A., Arenas-Sánchez, A., Alonso-Alonso, C., López-Heras, I., Nozal, L., RivasTabares, D., Vighi, M., 2019. Identification of contaminants of concern in the upper Tagus river basin (central Spain). Part 1: Screening, quantitative analysis and comparison of sampling methods. Sci. Total Environ. 666, 1058-1070. doi:10.1016/j.scitotenv.2019.02.250.

Saunders, D.L., Meeuwig, J.J., Vincent, A.C.J., 2002. Freshwater protected areas: strategies for conservation. Conserv. Biol. 16, 30-41. doi:10.1046/j.1523-1739. 2002.99562.x.

Schmidt, N., Fauvelle, V., Ody, A., Castro-Jiménez, J., Jouanno, J., Changeux, T., Thibaut, T., Sempéré, R., 2019. The amazon river: a major source of organic plastic additives to the tropical North Atlantic? Environ. Sci. Technol. 53, 7513-7521. doi:10.1021/acs.est.9b01585.

Schymanski, E.L., Jeon, J., Gulde, R., Fenner, K., Ruff, M., Singer, H.P., Hollender, J., 2014. Identifying small molecules via high resolution mass spectrometry: communicating confidence. Environ. Sci. Technol. 48, 2097-2098. doi:10.1021/ es5002105.

Shrivastava, M., Andreae, M.O., Artaxo, P., Barbosa, H.M.J., Berg, L.K., Brito, J., Ching, J., Easter, R.C., Fan, J., Fast, J.D., Feng, Z., Fuentes, J.D., Glasius, M., Goldstein, A.H., Alves, E.G., Gomes, H., Gu, D., Guenther, A., Jathar, S.H., Kim, S., Liu, Y., Lou, S., Martin, S.T., McNeill, V.F., Medeiros, A., de Sá, S.S., Shilling, J.E., Springston, S.R., Souza, R.A.F., Thornton, J.A., Isaacman-VanWertz, G., Yee, L.D. Ynoue, R., Zaveri, R.A., Zelenyuk, A., Zhao, C., 2019. Urban pollution greatly enhances formation of natural aerosols over the Amazon rainforest. Nat. Commun. 10, 1046. doi:10.1038/s41467-019-08909-4.

SNIS. Brazilian National System of Information about the sanitation system, 2020 . Indicators for water supply and sewage. [WWW Document]. URL http://www. snis.gov.br/ (accessed 9.23.20).

Neto, Sotão, B.M.T., Combi, T., Taniguchi, S., Albergaria-Barbosa, A.C.R., Ramos, R.B., Figueira, R.C.L., Montone, R.C., 2020. Persistent organic pollutants (POPs) and personal care products (PCPs) in the surface sediments of a large tropical bay (Todos os Santos Bay, Brazil). Mar. Pollut. Bull. 161, 111818. doi:10.1016/j. marpolbul.2020.111818.

Tedesco, P.A., Beauchard, O., Bigorne, R., Blanchet, S., Buisson, L., Conti, L., Cornu, J.F., Dias, M.S., Grenouillet, G., Hugueny, B., Jézéquel, C., Leprieur, F., Brosse, S., Oberdorff, T., 2017. A global database on freshwater fish species occurrence in drainage basins. Sci. Data 4, 170141. doi:10.1038/sdata.2017.141.

Thomas, K.V., da Silva, F.M.A., Langford, K.H., de Souza, A.D.L., Nizzeto, L., Waichman, A.V., 2014. Screening for selected human pharmaceuticals and cocaine in the urban streams of manaus, Amazonas, Brazil. JAWRA J. Am. Water Resour. Assoc. 50, 302-308. doi:10.1111/jawr.12164.

Tian, Z., Peter, K.T., Gipe, A.D., Zhao, H., Hou, F., Wark, D.A., Khangaonkar, T., Kolodziej, E.P., James, C.A., 2020. Suspect and nontarget screening for contaminants of emerging concern in an urban estuary. Environ. Sci. Technol. 54, 889901. doi:10.1021/acs.est.9b06126.

Tisseuil, C., Cornu, J.-F., Beauchard, O., Brosse, S., Darwall, W., Holland, R Hugueny, B., Tedesco, P.A., Oberdorff, T., 2013. Global diversity patterns and 
cross-taxa convergence in freshwater systems. J. Anim. Ecol. 82, 365-376. doi:10.1111/1365-2656.12018.

Valdez-Carrillo, M., Abrell, L., Ramírez-Hernández, J., Reyes-López, J.A., CarreónDiazconti, C., 2020. Pharmaceuticals as emerging contaminants in the aquatic environment of Latin America: a review. Environ. Sci. Pollut. Res. 27, 4486344891. doi:10.1007/s11356-020-10842-9. van der Aa, M., Bijlsma, L., Emke, E., Dijkman, E., van Nuijs, A.L.N., van de Ven, B., Hernández, F., Versteegh, A., de Voogt, P., 2013. Risk assessment for drugs of abuse in the Dutch watercycle. Water Res 47, 1848-1857. doi:10.1016/j.watres. 2013.01.013. 